\title{
Plastic bottle oscillator: Rhythmicity and mode bifurcation of fluid flow
}

$\operatorname{AUTHOR}(\mathrm{S})$ :

Kohira, Masahiro I.; Magome, Nobuyuki; Kitahata, Hiroyuki; Yoshikawa, Kenichi

\section{CITATION:}

Kohira, Masahiro I. ... [et al]. Plastic bottle oscillator: Rhythmicity and mode bifurcation of fluid flow. American Journal of Physics 2007, 75(10): 893-895

ISSUE DATE:

2007-10

URL:

http://hdl.handle.net/2433/49730

\section{RIGHT:}

Copyright (2007) American Association of Physics Teachers. This article may be downloaded for personal use only. Any other use requires prior permission of the author and the American Association of Physics Teachers; この論文は出版社 版でありません。引用の際には出版社版をご確認ご利用ください。; This is not the published version. Please cite only the published version. 


\title{
Plastic bottle oscillator: Rhythmicity and mode bifurcation of fluid flow
}

\author{
Masahiro I. Kohira \\ Japan Space Forum, Otemachi, Chiyoda-ku, Tokyo 100-0004, Japan \\ Nobuyuki Magome \\ Department of Food and Nutrition, \\ Nagoya Bunri College, Nagoya 451-007r, Japan \\ Hiroyuki Kitahata and Kenichi Yoshikawa* \\ Department of Physics, Graduate School of Science, \\ Kyoto University 85 Spatiotemporal Project, \\ ICORP, JST, Kyoto 606-8502, Japan
}

\begin{abstract}
The oscillatory flow of water draining from an upside-down plastic bottle with a thin pipe attached to its head is studied as an example of a dissipative structure generated under far-fromequilibrium conditions. Mode bifurcation was observed in the water/air flow: no flow, oscillatory flow, and counter flow were found when the inner diameter of the thin pipe was changed. The modes are stable against perturbations. A coupled two-bottle system exhibits either in-phase or anti-phase self-synchronization. These characteristic behaviors imply that the essential features of this oscillatory flow in a single bottle system should be described as a limit-cycle oscillation.
\end{abstract}




\section{INTRODUCTION}

The natural world presents a rich variety of dissipative structures where the interactions between the constituent elements produces spatio-temporal order. Examples include heart beats $^{1}$, flashing fireflies, ${ }^{2,3}$ hand clapping in the theater ${ }^{4}$, and price movements in the stock market $^{5}$. To understand these phenomena, theoretical and numerical studies on coupled nonlinear oscillators have been extensively performed. ${ }^{4,6-9}$ Experimental studies of instabilities in fluid systems are of interest in Benard convection and Lorenz model. ${ }^{10-17}$

We report on a novel rhythmic phenomenon involving water/air flow caused by gravitational instability. A plastic bottle with a thin pipe attached to its head is used (see Fig. 1). When the inner diameter of the pipe is changed, bifurcation among three modes is observed: no flow, oscillatory flow, and counter flow. When a coupled system is constructed with a pair of bottles, the oscillators are entrained to each other and are in either in- or anti-phases depending on the coupling.

Periodic switching occurs between the downflow of water and the upflow of air in the plastic bottle oscillator. The asymmetric features of the periodic flow can be interpreted in terms of the parameters that characterize the motion of the water and the air moving through the pipe. Instead of a detailed theory, we propose a model equation by focusing on the limit-cycle oscillation behavior. The simple model may be useful for increasing student understanding and the similarity with many other oscillatory phenomena in nature, such as various biological phenomena ${ }^{3,18,19}$ and electrical circuits ${ }^{4}$.

\section{EXPERIMENTS}

A thin acrylic pipe (length $\ell=100 \mathrm{~mm}$, inner diameter $d$ such that $2 \mathrm{~mm} \leq d \leq 24 \mathrm{~mm}$ ) was attached to the head of a plastic bottle made of poly-ethylene terephthalate (PET), which was obtained commercially as a water bottle containing $2 \mathrm{~L}$ of liquid. The bottle was filled with $1.8 \mathrm{~L}$ of water and placed upside down as shown in Fig. 1(a). The time-dependent changes in the system were monitored with a digital video camera, recorded on videotape, and analyzed by an image-processing system. ${ }^{20}$ To help visualize the flow, a few drops of black ink were mixed into the water.

Figure 2 summarizes the experimental observations of the flow modes in a single oscillator, 
which are given in terms of the inner diameter $d$ of the thin pipe. Just after the bottle is placed upside down, the water in the bottle starts to drain through the pipe. When $d$ is equal to or less than $4 \mathrm{~mm}$, the downflow of water is retarded and stopped, a no flow state. When $d$ is between $6 \mathrm{~mm}$ and $16 \mathrm{~mm}$, periodic switching between the downflow of water and upflow of air is observed. This switching continues until all of the water in the bottle is gone; the period of oscillation gradually increases as the amount of water inside the bottle decreases. Figure 1(b) shows a spatio-temporal diagram of the oscillation that is observed in this region. This oscillatory flow is stable and resumes after a strong forcing is applied, such as shaking the bottle or stopping the flow for some time. The period of oscillation tends to decrease as the inner diameter of the pipe is increased. When $d$ was equal to or greater than $20 \mathrm{~mm}$, the counter flow of water/air is observed. This flow continues until no water remains.

Figure 3 shows the experimental results for a coupled two-bottle system. When two bottles with almost the same oscillatory flow condition are interconnected by a hollow tube between the air spaces of each bottle, they exhibit in-phase synchronized oscillation. When they were interconnected by a U-shaped tube containing water between the air spaces of each bottle, they exhibit anti-phase synchronized oscillation. These two modes are stable despite the gradual change in the period due to the decrease in the water level, and resume after the application of a perturbation.

\section{DISCUSSION}

We now discuss the mechanism of the oscillatory phenomenon we have described. Just after the start of the experiment, the water inside the single bottle system begins to move downward through the pipe due to gravity. As the downflow progresses, the gauge pressure inside the bottle gradually increases, which tends to retard the flow. In the region of no flow $(d \leq 4 \mathrm{~mm})$, the inertia of the downflow is not large enough to reverse the pressure difference, and thus the downflow of water terminates and a steady state without flow is achieved. For oscillatory flow $(6 \mathrm{~mm} \leq d \leq 16 \mathrm{~mm})$, the damping effect of the thin pipe is less than that in the no flow region and transient flow is observed for a while after the total pressure reaches equilibrium. When the downflow of water stops as a result of damping, the pressure difference is reversed and the upflow of air is induced. The same type of transient 
flow occurs for the upflow of air, and the downflow of water then recurs. For counter flow $(d \geq 20 \mathrm{~mm})$, the inner diameter of the pipe is so large that the downflow of water and upflow of air can occur simultaneously against friction and viscosity.

The three modes can also be seen when a glass bottle is used instead of a plastic bottle, but a plastic bottle is better for observing the oscillatory flow because of the elasticity of the bottle. "Tornado in a bottle" is a well-known experiment in meteorology by creating a vortex in the outgoing water by rotating the bottle around a vertical axis ${ }^{21}$. The coupling between this vortex and the periodic phenomenon may be an interesting extension of the experiments using the plastic bottle oscillator.

Next, let us discuss the mechanism of synchronization in the coupled two-bottle system. When two bottles are interconnected by a hollow tube, the air pressure inside the bottles is the same because the relaxation time of the pressure difference is small relative to the period of oscillation. Therefore, the interaction between the bottles reflects the tendency for the pressure difference to stay small. When the two bottles are interconnected by a U-shaped tube containing water, the air spaces of the bottles are separated by the water in the tube. If there is a phase difference between the bottles, the water in the interconnecting tube moves to reduce the pressure difference between the two bottles. Thus, the water in the tube serves to increase the compressibility of air space in each bottle, and the interaction between the bottles has the opposite sign to that in the case of the hollow tube.

The unique characteristics of the plastic bottle oscillation is the occurrence of sustained rhythmic flow. This rhythmic flow is the periodic alternation of the two different states of flow, upflow of air and downflow of water. Instead of a detailed interpretation of the equation of motion, we note that the sustained rhythm can be interpreted by connecting two different equations of motion. Consider the following model equation:

$$
\rho \frac{\mathrm{d}^{2} x}{\mathrm{~d} t^{2}}+\mu \frac{\mathrm{d} x}{\mathrm{~d} t}+\omega^{2} x=a \operatorname{sgn}\left(\frac{\mathrm{d} x}{\mathrm{~d} t}\right)
$$

where $\operatorname{sgn}(\beta)=1,0,-1$ for $\beta>1, \beta=0$, and $\beta<0$, respectively, $\rho$ is the density of water, $\mu$ is the reciprocal of the inner diameter of the thin pipe, $\omega$ is the sum of the compressibility of air and the elasticity of the bottle, and $a$ is a positive constant which is related to the gravity and buoyancy. Equation (1) can reproduce the features observed in the plastic bottle oscillator, such as limit-cycle oscillation, bifurcation, and synchronization. 


\section{CONCLUSION}

Mode bifurcation and the limit-cycle oscillation of water/air flow were observed in a simple experiment using a plastic bottle, a thin acrylic pipe, and water. The bifurcation can be controlled by changing the inner diameter of the thin pipe attached to the head of the bottle. The limit-cycle oscillation of water/air was stable within a wide range of the inner diameter of the thin pipe. In the coupled system, two kinds of synchronization, inand anti-phase modes, were observed experimentally with changes in the coupling. The experimental results suggest that a rich variety of interesting modes will appear for coupled systems with more than two bottles. We stress that we can easily choose the experimental parameters so as to make the synchronization between a pair of oscillators either in-phase or anti-phase. For example, it is possible to construct a coupled oscillator with frustration if we consider the analogy to the ferro and anti-ferro magnetic interactions between a pair of spins. ${ }^{22}$

\section{Acknowledgments}

The authors would like to thank Dr. Shin-ichiro M. Nomura (Tokyo Medical and Dental University, Japan) and Dr. Ryoichi Aihara (West Virginia University) for their kind advice. This work was supported in part by Kurata Grants from the Kurata Memorial Hitachi Science and Technology Foundation and a Grant-in-Aid for Scientific Research in Priority Areas (No. 17049017) from the Ministry of Education, Culture, Sports, Science and Technology of Japan to H. K.

* To whom correspondence should be addressed. Tel:+81-75-753-3812. Fax:+81-75-753-3779. Email: yoshikaw@scphys.kyoto-u.ac.jp

1 L. Glass, M. R. Guevara and A. Shrier, "Bifurcation and Chaos in a Periodically Stimulated Cardiac Oscillator", Physica D 7, 89-101 (1983).

2 H. M. Smith, "Synchronous Flashing of Fireflies", Science 82, 151-152 (1935).

3 S. H. Strogatz, Sync: The Emerging Science of Spontaneous Order (Theia, New York, 2003). 
4 A. Pikovsky, M. Rosenblum, and J. Kurths, Synchronization: a Universal Concept in Nonlinear Sciences (Cambridge University Press, Cambridge, 2001).

5 R. H. Day and P. Chen, Nonlinear Dynamics and Evolutionary Economics (Oxford University Press, New York, 1993).

6 A. T. Winfree, The Geometry of Biological Time (Springer, New York, 1980).

7 H. Haken, Synergetics (Springer, Berlin, 1977).

8 G. Nicolis and I. Prigogine, Self-Organization in Nonequilibrium Systems (Wiley \& Sons, New York, 1977).

9 Y. Kuramoto, Chemical Oscillations, Waves, and Turbulence (Springer, Berlin, 1984).

10 M. C. Cross and P. C. Hohenberg, "Pattern-Formation outside of Equilibrium", Rev. Mod. Phys. 65, 851-1112 (1993).

11 E. N. Lorenz, The Essence of Chaos (University of Washington Press, Seattle, 1993).

12 K. Yoshikawa, N. Oyama, M. Shoji, and S. Nakata, "Use of a Saline Oscillator as a Simple Nonlinear Dynamic System - Rhythms, Bifurcation, and Entrainment", Am. J. Phys. 59, 137$141(1991)$.

13 M. Okamura and K. Yoshikawa, "Rhythm in a Saline Oscillator", Phys. Rev. E 61, 2445-2452 (2000).

14 H. Kitahata, A. Yamada, and S. Nakata, "Mode Bifurcation by Pouring Water into a Cup", J. Chem. Phys. 119, 4811-4816 (2003).

15 C. Clanet and G. Searby, "On the Glug-glug of Ideal Bottles", J. Fluid Mech. 510, 145-168 (2004).

16 R. Shaw, The Dripping Faucet as a Model Chaotic System (Aerial Press, Santa Cruz, 1984).

17 C. Ellegaard, A. E. Hansen, A. Haaning, K. Hansen, A. Marcussen, T. Bohr, J. L. Hansen, and S. Watanabe, "Creating Corners in Kitchen Sinks", Nature 392, 767-768 (1998).

18 I. Aihara, S. Horai, H. Kitahata, K. Aihara, and K. Yoshikawa, "Dynamical Calling Behaviors Experimentally Observed in Japanese Tree Frogs (Hyla japonica)", IEICE Transactions, in press.

19 A. Takamatsu, R. Tanaka, H. Yamada, T. Nakagaki, T. Fujii. and I. Endo, "Spatio-temporal Symmetry in Rings of Coupled Biological Oscillators of Physarum plasmodium", Phys. Rev. Lett. 87 078102, 1-4 (2001).

20 We used ImageJ, 〈http://rsb.info.nih.gov/ij/〉. 
21 E. D. Schneider and D. Sagan, Into the Cool (The University of Chicago Press, Chicago, 2005).

22 M. Yoshimoto, K. Yoshikawa, and Y. Mori, "Coupling among 3 Chemical Oscillators - Synchronization, Phase Death, and Frustration", Phys. Rev. E 47, 864-874 (1993). 


\section{Figure Captions}

(a)

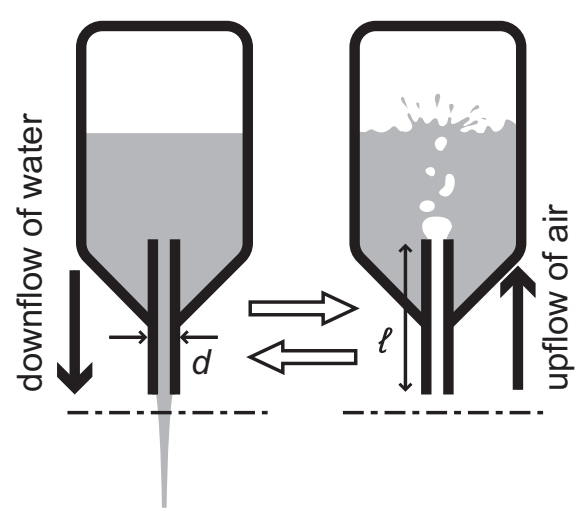

(c)

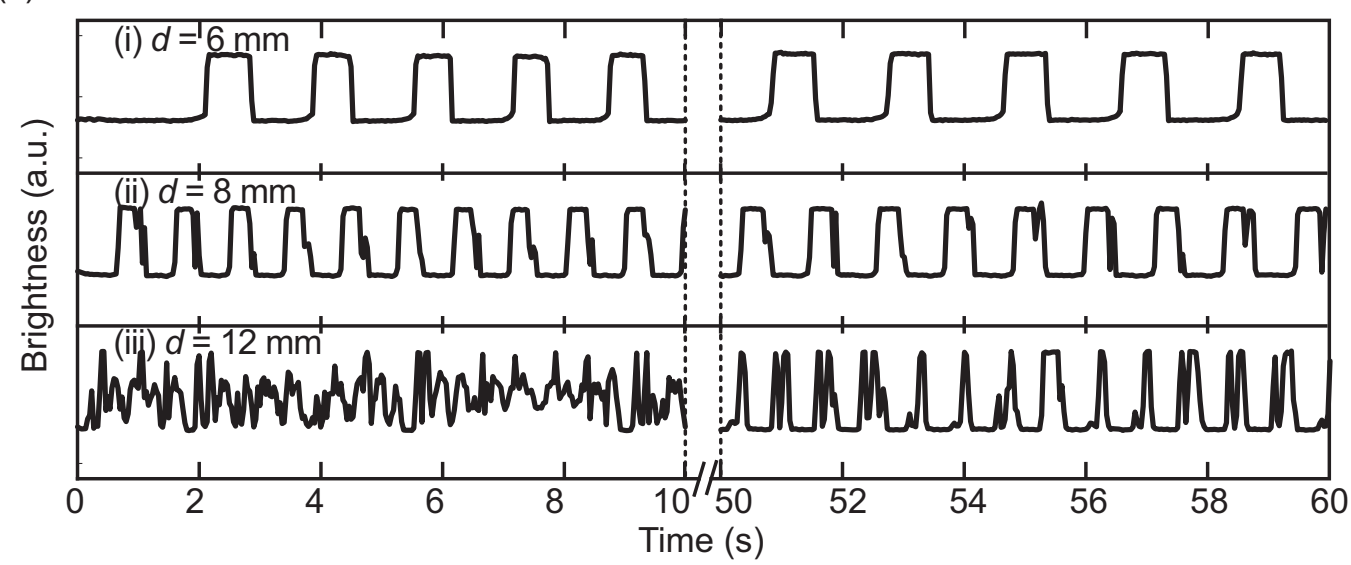

FIG. 1: (a) Schematic representation of the plastic bottle oscillator. The repetitive upflow of air and downflow of water through the thin pipe were observed. (b) Spatio-temporal diagram of the plastic bottle oscillation depicted by aligning the horizontal images $3 \mathrm{~mm}$ below the lower end of the thin pipe (along the dash-dotted line shown in (a)) with a time series. Dark and bright regions correspond to the downflow of water and the upflow of air, respectively. (c) Time trace of water flow, as represented by the brightness in the central part of the image in (b). Positive and negative values correspond to upflow and downflow, respectively. The length of the thin pipe was $100 \mathrm{~mm}$, and the inner diameters of the thin pipe were (i) $6 \mathrm{~mm}$, (ii) $8 \mathrm{~mm}$, and (iii) $12 \mathrm{~mm}$, respectively. The time $t=0$ corresponds to the moment when the water was allowed to flow by opening the lower end of the thin pipe. 


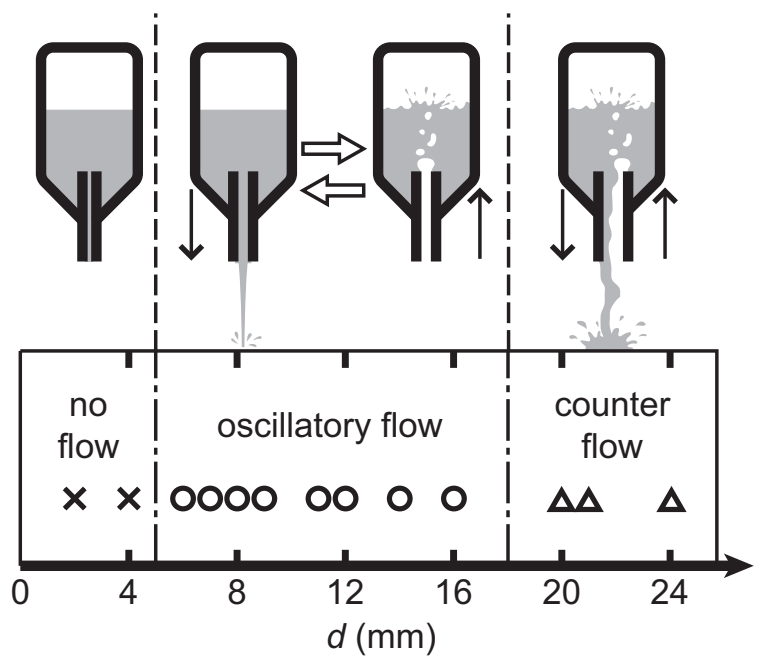

FIG. 2: Mode bifurcation of the flow as a function of the inner diameter of the thin pipe at the head of the bottle. The cross, circle, and triangle correspond to no flow, oscillatory flow, and counter flow, respectively. The length of the thin pipe was $100 \mathrm{~mm}$. 
(a)

(i)

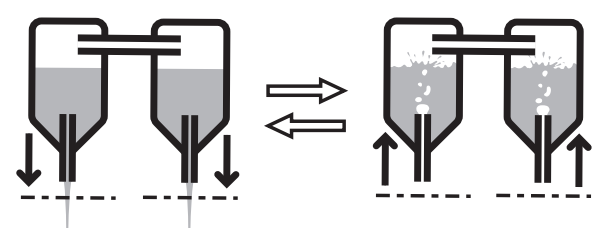

(ii)

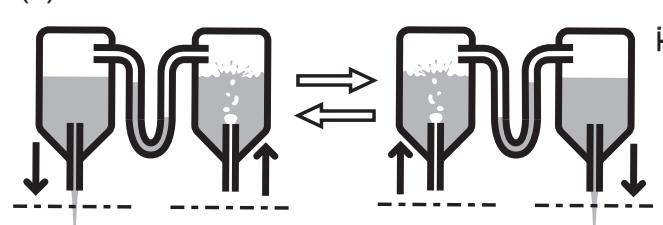

(c)

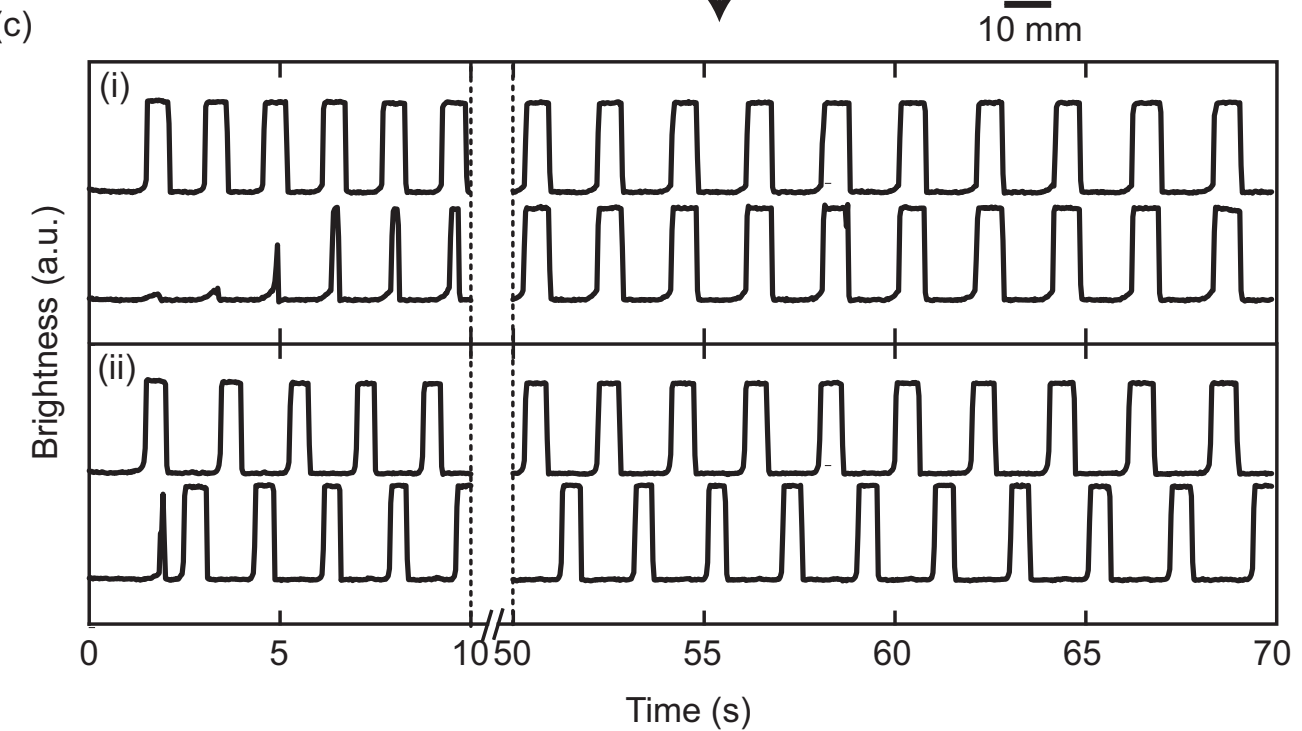

FIG. 3: Experimental results for the synchronization of the two plastic bottle oscillators. (a) Schematic illustration of the coupled oscillators. (b) Spatio-temporal diagrams generated in the same way as in Fig. 1(b). (c) Time series of the oscillators. The high and low brightness correspond to the downflow of water and upflow of air, respectively. (i) The bottles were interconnected by a hollow tube (length of tube $\ell=1000 \mathrm{~mm}$, inner diameter of tube $d=8 \mathrm{~mm}$ ). The phases of the two oscillators are almost the same, that is, they exhibited in-phase synchronization. (ii) The bottles are interconnected by a U-shaped tube containing water $(\ell$ and $d$ are the same as (i), and the amount of water was $10 \mathrm{~mL}$ ). The phase difference between the two oscillators is around $\pi$, that is, they exhibited anti-phase synchronization. The plastic bottles are the same as those used in Fig. 2(a). The inner diameters of the thin pipes at the head of the bottle were $6 \mathrm{~mm}$. 